Mechanisms of resonant low frequency Raman scattering from metallic nanoparticle Lamb modes

A. Girard, J. Lermé, H. Gehan, J. Margueritat, and A. Mermet

Citation: The Journal of Chemical Physics 146, 194201 (2017); doi: 10.1063/1.4983119

View online: http://dx.doi.org/10.1063/1.4983119

View Table of Contents: http://aip.scitation.org/toc/jcp/146/19

Published by the American Institute of Physics

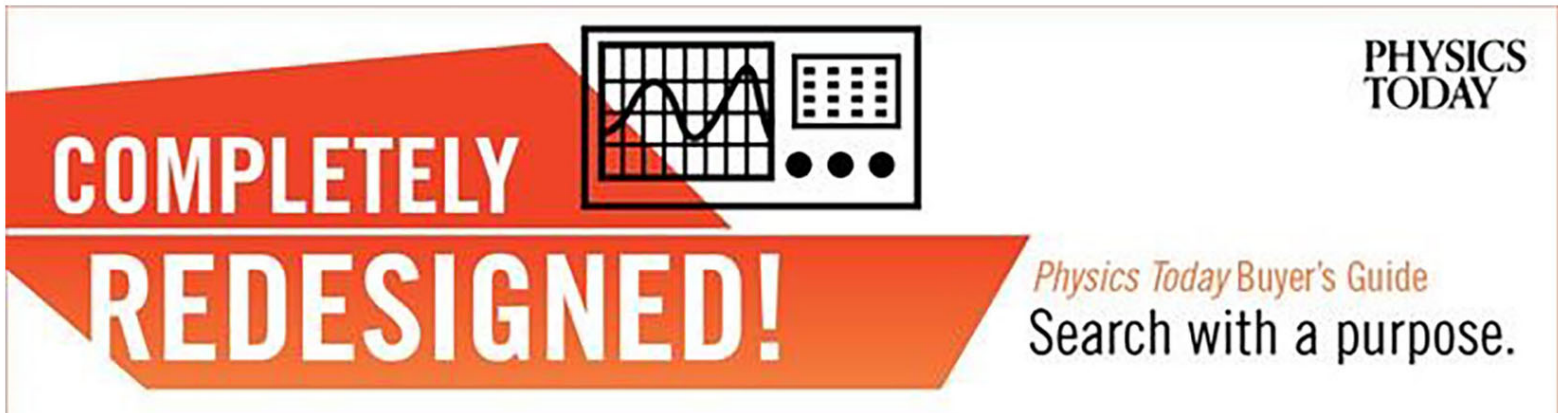




\title{
Mechanisms of resonant low frequency Raman scattering from metallic nanoparticle Lamb modes
}

\author{
A. Girard, J. Lermé, H. Gehan, J. Margueritat, and A. Mermet \\ Institut Lumière Matière, Université de Lyon, Université Claude Bernard Lyon 1, UMR CNRS 5306, 69622 \\ Villeurbanne, France
}

(Received 11 January 2017; accepted 25 April 2017; published online 16 May 2017)

\begin{abstract}
The low frequency Raman scattering from gold nanoparticle bimodal assemblies with controlled size distributions has been studied. Special care has been paid to determining the size dependence of the Raman intensity corresponding to the quadrupolar Lamb mode. Existing models based on a microscopic description of the scattering mechanism in small particles (bond polarizability, dipole induced dipole models) predict, for any Raman-active Lamb modes, an inelastic intensity scaling as the volume of the nanoparticle. Surprisingly experimental intensity ratios are found to be anomalously much greater than theoretical ones, calling into question this scaling law. To explain these discrepancies, a simple mechanism of Raman scattering, based on the density fluctuations in the nanoparticles induced by the Lamb modes, is introduced. This modeling, in which the nanoparticle is described as an elastic isotropic continuous medium - as in Lamb theory, successfully explains the major features exhibited by low frequency Raman modes. Moreover this model provides a unified picture for any material, suitable for handling both small and large size ranges, as well as non-resonant and resonant excitation conditions in the case of metallic species. Published by AIP Publishing. [http://dx.doi.org/10.1063/1.4983119]
\end{abstract}

\section{INTRODUCTION}

Low frequency (LF) Raman scattering is a widely used approach to experimentally investigate the vibrational dynamics of nanomaterials. In the past decades, special attention was paid to localized acoustic modes, also called Lamb modes, because they can provide valuable information about the morphology, ${ }^{1-3}$ the surface state, ${ }^{4}$ and the crystallinity ${ }^{5,6}$ of the probed nano-objects. Mostly metallic, ${ }^{5,7-9}$ dielectric, ${ }^{10,11}$ and semiconducting ${ }^{12,13}$ spherical nanoparticles (NPs) have been studied but recent advances in chemistry-based synthesis have allowed us to consider other geometries of confinement such as nanoplates,${ }^{4,14,15}$ nanobipyramids, ${ }^{16}$ or nanorods ${ }^{17,18}$ with low size dispersion. Most useful information from Lamb modes is usually obtained by analysing the position and width of the LF Raman peaks ${ }^{19,20}$ while less attention is paid to their intensities which are much harder to interpret due to intercompetition between several processes (multiple scattering, resonance conditions, re-absorption).

The theoretical description of the inelastic light scattering mechanisms by Lamb modes has been subject to a longstanding discussion in the past ${ }^{21-24}$ regarding the attribution of the experimentally observed modes and the establishment of selection rules, first established by Duval, ${ }^{25}$ which predict that the LF spectra of small nanoparticles remain exclusively dominated by breathing (angular momentum $\ell=0$ ) and quadrupolar modes $(\ell=2)$. The topic has remained controversial until the introduction by Montagna et al. of a full theoretical modeling based on a microscopic description of the polarisability fluctuations generated by the vibrations that unambiguously explain which modes are experimentally revealed in the case of non-resonant scattering experiments. ${ }^{26-28}$ For small sizes compared to the optical wavelength, the particle behaves as a molecule and the scattering is described by dipole-induceddipole (DID) and bond polarisability (BP) models, and only quadrupolar and breathing modes are active. ${ }^{26}$ However the LF spectra of larger particles with a size comparable to the light wavelength contain significant contribution from higher $\ell$ modes. ${ }^{27,28}$ It is worthwhile stressing that the density fluctuations (i.e., the Brillouin mechanism) are not directly responsible for the emergence of Lamb modes with high $\ell$ and $n$ values in LF spectra (where $\mathrm{n}$ is the harmonic number). Indeed, the Brillouin mechanism being assumed to be active only in large particles, the modeling requires introducing retardation effects through the factor $e^{i \mathbf{q} \cdot \mathbf{r}}$, where $\mathrm{q}$ is the exchanged wavevector. This extra factor, which differs from unity for large enough sizes, is the direct cause of the emergence of high $\ell$ values. These models have been proven to successfully reproduce experimental spectra of both small and large particles. One advancement of our model compared to existing theories is the taking into account of the dependence of the scattering amplitude towards the internal electric field, which intervenes as a scaling factor, when tuning the optical wavelength in and out of resonance with the surface plasmon.

Metallic nanoparticles show strong absorption in the visible range due to the surface plasmon. In LF Raman experiment, tuning the excitation wavelength in resonance with the surface plasmon is of great benefit as it allows a considerable enhancement of the inelastic signal from Lamb modes. ${ }^{5,7-9}$ Moreover, the interest in understanding the mechanisms of inelastic scattering by metallic nanoparticle vibrations is still growing with the recent discovery of dimer vibrations from Au NPs that 
arise in the LF Raman spectra upon resonant excitation of the plasmonic modes of dimer Au NPs. ${ }^{29}$ The importance of the surface plasmon in LF Raman experiments is demonstrated by the high number of publications in previous years focusing on the acoustic properties of metallic nano-objects. However, despite this growing interest, a simple theoretical approach for modeling the Raman scattering from Lamb modes when the excitation light is tuned in resonance with the surface plasmon is still lacking.

In the present work, we experimentally investigate the inelastic scattering from Au NP Lamb modes using resonant LF Raman spectroscopy, with special focus on the NP volume dependence of the Raman intensity, that is theoretically predicted as a power law $I \propto V_{N P}$ by the microscopic model of Montagna et al. To the best of our knowledge this power law has not yet been experimentally verified. In order to quantitatively investigate this scaling law, we have studied NP bimodal assemblies with varying volumic proportions. Our results point out significant differences with the predictions from microscopic theory for non-resonant scattering. In order to explain the observed discrepancies, a simple mechanism of Raman scattering is introduced, based on density fluctuations in the NPs induced by the Lamb modes, that provides a unified picture for any material, suitable for handling both small and large size ranges, as well as non-resonant and resonant excitation conditions in the case of metallic species.

\section{EXPERIMENT}

The bimodal assemblies are constituted of two NP populations with diameters $\mathrm{D}=18 \mathrm{~nm}$ and $\mathrm{D}=49 \mathrm{~nm}$, respectively, referred to as Au18 and Au49 in the following. NPs were synthesized following a method already reported in the literature, see Ref. 29 for details. Polyvinyl pyrrolidone (PVP) has been
TABLE I. Main characteristics of several bimodal assemblies with the increasing volumic fraction of Au49 NPs from bottom to top (see text for details). NP Au(i) represents the number of NPs contained within each batch ( $i=18$ or $\mathrm{i}=49$ ) and $\% \mathrm{~N} \mathrm{Au}(\mathrm{i})$ represents the NP number fraction of population i over the total number of NPs.

\begin{tabular}{|c|c|c|c|c|c|c|}
\hline $\mathrm{x}(\%)$ & $\begin{array}{c}\text { NP } \\
\mathrm{Au} 18\end{array}$ & $\begin{array}{c}\mathrm{NP} \\
\mathrm{Au} 49\end{array}$ & $\begin{array}{c}\% \mathrm{~N} \\
\mathrm{Au} 18\end{array}$ & $\begin{array}{c}\% \mathrm{~N} \\
\mathrm{Au} 49\end{array}$ & $\begin{array}{c}\% \text { N Au } 18 \\
\text { TEM }\end{array}$ & $\begin{array}{c}\% \mathrm{~N} A u 49 \\
\text { TEM }\end{array}$ \\
\hline 100 & 0 & & 0 & 100 & & \\
\hline 84 & $8.36 \times 10^{10}$ & $2.21 \times 10^{10}$ & 79.1 & 20.9 & 82 & 18 \\
\hline 78 & $8.36 \times 10^{10}$ & $1.48 \times 10^{10}$ & 85 & 15 & & \\
\hline 70 & $8.36 \times 10^{10}$ & $9.9 \times 10^{9}$ & 89.4 & 10.6 & 90 & 10 \\
\hline 60 & $8.36 \times 10^{10}$ & $6.34 \times 10^{9}$ & 93 & 7 & 92 & 8 \\
\hline 39 & $8.36 \times 10^{10}$ & $2.61 \times 10^{9}$ & 97 & 3 & & \\
\hline 16 & $8.36 \times 10^{10}$ & $7.78 \times 10^{8}$ & 99.1 & 0.9 & & \\
\hline 7 & $8.36 \times 10^{10}$ & $3.01 \times 10^{8}$ & 99.6 & 0.4 & 99 & 1 \\
\hline 0 & $8.36 \times 10^{10}$ & 0 & 100 & 0 & & \\
\hline
\end{tabular}

used to ensure a good stabilization of the NPs. Then the different bimodal assemblies were obtained by mixing these two nanoparticle batches in their solvent with various relative concentrations before deposition on an indium tin oxide substrate for Raman characterization. Upon drying, the PVP forms a continuous film of approximative thickness $100 \mu \mathrm{m}$ in which NPs are embedded. The number of Au18 nm NPs is kept constant to $8.36 \times 10^{10}$ particles while the number of Au49 nm NPs is progressively increased from $3.01 \times 10^{8}$ to $2.21 \times 10^{10}$ in order to vary the relative volumic proportions. The details of intermediate batches are summarized in Table I, where $x$ represents the volumic fraction of Au49 NPs (conversely 1- $x$ for Au18). For consistency, we have realized a statistical study of four bimodal assemblies to cross-check the correctness of the estimated population ratios (batches $x=84 \%$, $x=70 \%, x=60 \%$, and $x=7 \%$ ). To this end, diluted solutions
Au18
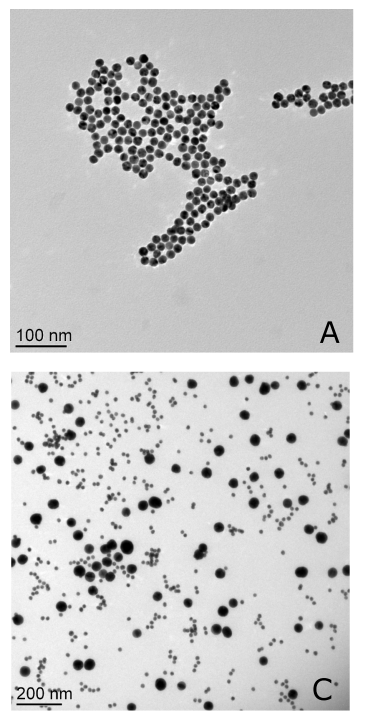

$x=78 \%$
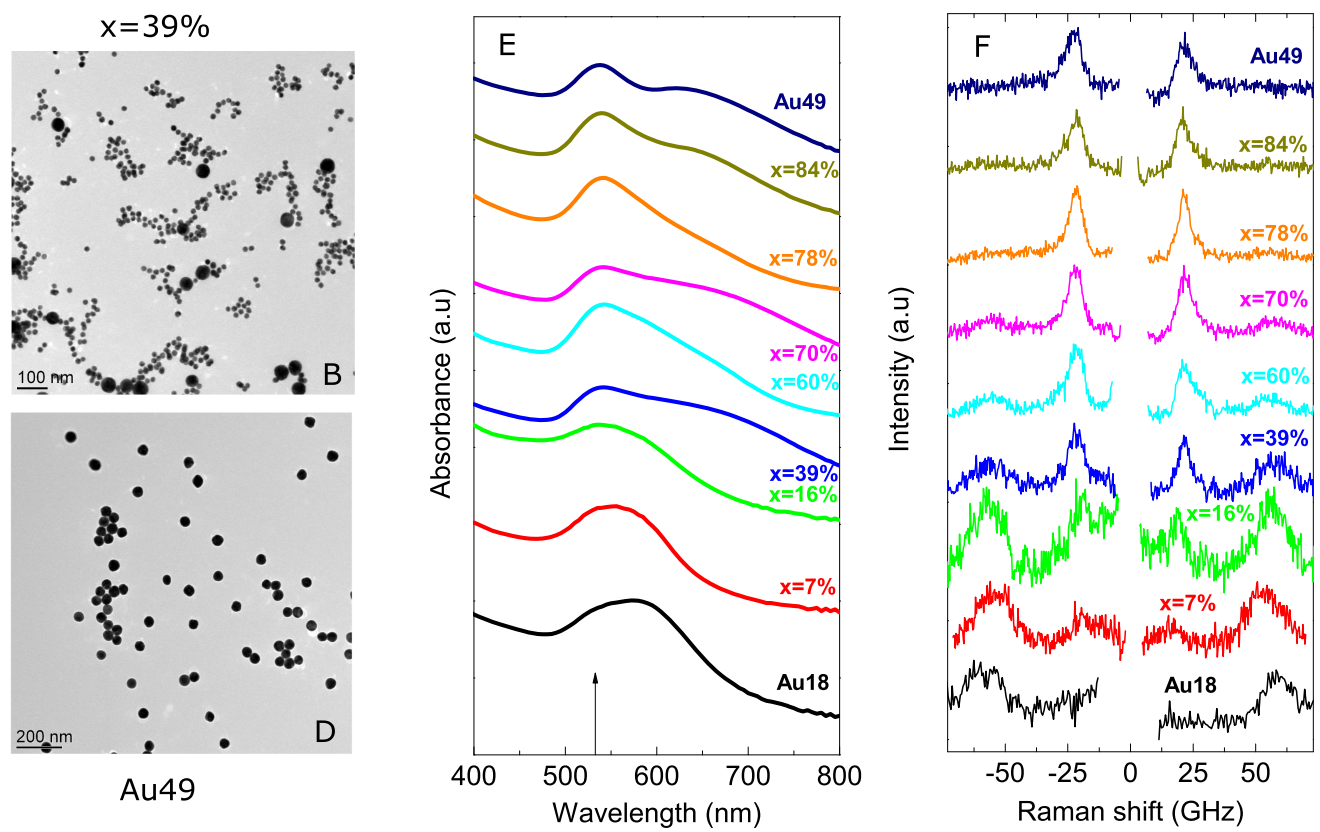

FIG. 1. ((a)-(d)) TEM images of Au18, Au49, and two bimodal assemblies with varying compositions of Au49 NPs. ((e) and (f)) Absorption and Raman spectra (respectively) of dry PVP films containing gold NPs for Au49 and Au18 samples and various intermediate bimodal assemblies. Absorbance and Raman intensities are expressed in a.u. and are vertically shifted for clarity. For Raman, excitation wavelength is indicated as an arrow. 
of NPs have been dropped onto microscopy grids and imaged using a Philips CM120 transmission electron microscope (see Figs. 1(a)-1(d)). TEM statistics of the NP number from both populations are summarized in Table I and agree with the estimated ones within $1 \%$.

In order to correlate the plasmonic and acoustical resonances of the NPs, absorption and Raman measurements from bimodal assemblies were performed on the dry films at the same position. Absorption spectra are measured in transmission configuration. The white light beam was focused onto the sample surface using a condenser with a numerical aperture of 0.65 , and the transmitted light was collected with a $100 \times$ objective $(\mathrm{NA}=0.95)$ and focused onto the entrance slit of a spectrometer equipped with a thermoelectric-cooled CCD. Then the low-frequency Raman spectra were collected in backscattering geometry using a $100 \times$ objective $(\mathrm{NA}=0.95)$ and focused on the entrance hole of a six pass tandem Fabry-Perot interferometer.

\section{RESULTS AND DISCUSSION}

\section{A. Optical and Raman characterization}

Absorption spectra of the 9 films are summarized in Fig. 1(e) with the increasing volumic fraction of Au49 NPs from bottom to top. All assemblies show the most intense peak around $540 \mathrm{~nm}$ corresponding to the Surface Plasmon Resonance (SPR) of isolated nanoparticles. The size dependence of the dipolar resonance is negligibly small compared to the width of the peaks to be resolved. The broader redshifted shoulder corresponds to the plasmonic coupling between neighbouring $\mathrm{NPs}^{30-32}$ and its important width results from the large size dispersion of the NP aggregates inside the films. The position of the coupling band redshifts upon increasing the NP size and keeping a constant interparticle separation (few $\mathrm{nm}$ due to the PVP shell around the NPs), as illustrated by the difference between upper and lower absorption spectra (Au49 and Au18 samples, respectively). The $532 \mathrm{~nm}$ excitation wavelength for the Raman experiment is set in resonance with the dipolar peak (vertical arrow). Fig. 1(f) summarizes LF Raman spectra of Au18 nm (bottom) and Au49 nm (top). For samples containing only one size of NPs, one band due to the scattering by the quadrupolar vibration is observed. This vibration occurs at $59 \mathrm{GHz}$ for Au18 nm and $22 \mathrm{GHz}$ for Au49 nm, following the well known diameter-frequency relationship,

$$
v=0.85 \frac{c_{T}}{D}
$$

where $c_{T}=1250 \mathrm{~m} / \mathrm{s}$ is the transverse sound velocity of gold, $\mathrm{D}$ is the nanoparticle diameter, and 0.85 is a shape factor. Intermediate spectra correspond to the bimodal assemblies. As the volumic fraction of Au49 NPs is increased (bottom to top), Au49 quadrupolar intensity increases relatively to Au18 up to the threshold volume fraction $x_{\text {lim }}=78 \%$, above which the scattering from Au18 nm NP is not discernable anymore.

\section{B. Non-resonant LF Raman scattering: Microscopic model}

In the following, we compare the experimental intensity ratios, dependent on the volumic fraction $x$ of Au49 NPs, with the results of microscopic theory. The Raman intensity, for each Lamb mode, can be expressed as the product of a spatial term and a temporal term which corresponds, respectively, to the space- and time-Fourier components of the correlation function of the susceptibility fluctuations,

$$
I_{\text {Raman }}(a) \propto I_{s}(a) \times I_{t}(a),
$$

where the label $\mathrm{a} \equiv(\ell, \mathrm{n}, \mathrm{m})$ stands for any vibrational Lamb mode contributing to the inelastic Raman scattering. The temporal factor, $I_{t}(a)$, is given by (only the Stokes contribution is written below)

$$
\begin{aligned}
I_{t}(a) & \propto \int d t e^{-i \omega t} \frac{\hbar}{2 m \omega_{a}}\left(1+n\left(\omega_{a}\right)\right) e^{i \omega_{a} t} \\
& =\frac{h}{2 m \omega_{a}}\left(1+n\left(\omega_{a}\right)\right) \delta\left(\omega-\omega_{a}\right),
\end{aligned}
$$

where $n\left(\omega_{a}\right)$ is the Bose factor. Within the microscopic description of the polarisability fluctuations (Ref. 26), the Raman intensity can be written in the form

$$
I_{\text {Raman }}(a) \propto \frac{1+n\left(\omega_{a}\right)}{\omega_{a}} C\left(\omega_{a}\right),
$$

where $C(\omega)$ is the mode radiation coupling coefficient. Given that $\mathrm{n}(\omega)+1 \approx \mathrm{kT} / \hbar \omega$ at room temperature and $C(\omega) \propto 1 / \omega^{26}$ (from the microscopic model), one obtains the scaling law

$$
I_{\text {Raman }}(\omega) \propto \omega^{-3} \propto R^{3}
$$

where $\mathrm{R}$ is the NP radius. In a batch with Au49 volumic fraction $x, I_{\mathrm{Au} 49}^{\ell=2} \propto x V_{\mathrm{TOT}}$ and $I_{\mathrm{Au} 18}^{\ell=2} \propto(1-x) V_{\mathrm{TOT}}$, where $V_{\text {TOT }}$ is the volume of gold contained in the total number of NPs, and the intensity ratio between Au49 and Au18 quadrupolar modes scales as

$$
I_{A u 49}^{\ell=2} / I_{A u 18}^{\ell=2} \propto \frac{x}{1-x} .
$$

Experimental intensity ratios $I_{A u 49}^{\ell=2} / I_{A u 18}^{\ell=2}$ are evaluated from peaks top intensity after linear background subtraction and are Stokes/a-Stokes averaged. The comparison between the experimental ratios and the ones computed on the basis of microscopic theory is represented in Fig. 2. This figure clearly shows that the theoretical predictions underestimate the intensity of the bigger NPs with respect to the smaller ones (see

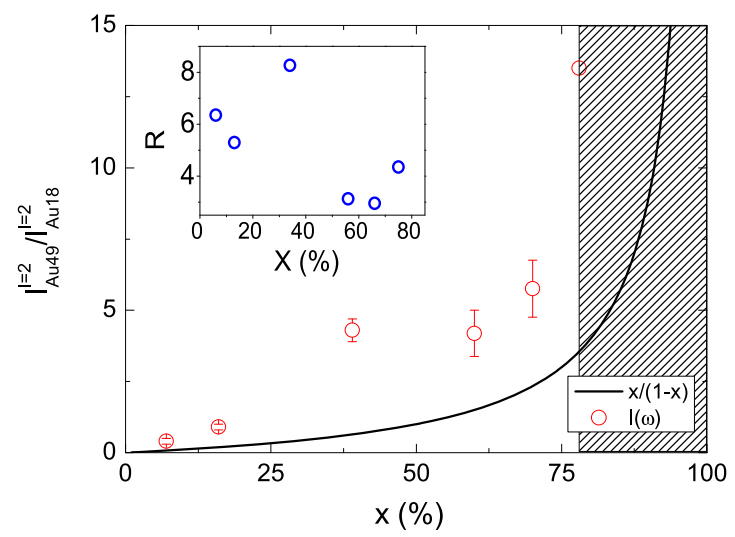

FIG. 2. Comparison between experimental quadrupolar mode intensity ratios of Au49 and Au18 NPs (red) for bimodal assemblies with various volumic compositions and theoretical predictions from non-resonant scattering theory. Inset: Ratio $\mathrm{R}$ between experimental and theoretical values. 
the inset, $\mathrm{R}$ is the ratio between experimental and theoretical values), pointing at an increased dependence of the scattered intensity toward the NP volume. It should be pointed out that the ratios (Eq. (6)) to be computed for the various composite samples are directly grounded on the linear law $I_{\text {Raman }} \propto V$ (Eq. (5)) which was established for a single scattering nanoparticle. In reality, clustering does occur in the PVP film, and it is likely that the proximity of neighbouring nanoparticles and clustering may affect the averaged response of the assembly. Nevertheless, we showed in a previous paper that the green wavelength $(532 \mathrm{~nm})$ is far enough from the plasmonic coupling band to avoid Raman measurements to be biased by the effects of nanoparticle clustering. In particular, we showed that Raman scattering is not sensitive to interaction effects for a resonant excitation of the dipolar surface plasmon, while coupled acoustic modes arise in the low frequency part of the Raman spectra for a red excitation $(647 \mathrm{~nm}){ }^{29}$ For this reason, we believe that the interaction effects within the clusters will not modify significantly the linear relationship in volume for this particular wavelength. To explain the discrepancies between experimental observations and microscopic model predictions, a simple mechanism of Raman scattering has been developed.

\section{LF Raman scattering: A continuous medium model}

In our model, the spatial- and time-dependent changes of the local susceptibility are assumed to result from the density fluctuations of the homogeneous NPs that are induced by the acoustic vibrations. This model will be described in great detail in a forthcoming paper so only the broad lines are reported in the present work. It is sufficient here to emphasize that the model is handled within a purely classical framework and is fully consistent with the Lamb theory (acoustic vibrations of an elastic and isotropic homogeneous sphere of density $\left.\rho_{0}\right) .{ }^{33,34}$ Indeed the model is built up from the basic equations of the classical radiating dipole theory, as the standard classical theories of elastic and inelastic scattering by molecules, liquids, and solids.

Before introducing the model, it is worth discussing briefly the size dependence $\left(\mathrm{I} \propto V_{N P}\right)$ that is predicted by existing microscopic atomistic models. ${ }^{26,28}$ Let us consider a homogeneous dielectric or metallic spherical scatterer (radius R, volume $\mathrm{V}$ ) in vacuum subject to an incoming monochromatic linearly polarized plane wave $\mathbf{E}(\mathbf{r}, t)=\mathbf{E}_{0} e^{i\left(\mathbf{k}_{0} \cdot \mathbf{r}-\omega_{0} t\right)}$ (the origin is taken at the sphere center). Assuming for simplicity the Born approximation (the local field is equal to the applied field), as assumed in practice in the Raman- and Brillouinrelated literature, ${ }^{26,28}$ the Rayleigh and inelastic scattering intensities at position $\mathrm{P}$ (located in the direction $\mathbf{u}$ ) and time $t$ are proportional to the square modulus of their respective corresponding scattered far-field and are given by

$$
\begin{gathered}
I_{\text {Rayleigh }}(P, t)=A \omega_{0}^{4}\left|\int_{V} \chi_{0} e^{i\left(\mathbf{k}_{\mathbf{0}}-\mathbf{k}\right) \cdot \mathbf{r}} d^{3} r\right|^{2}, \\
I_{\text {Inelastic }}(P, t)=A \omega_{0}^{4}\left|\int_{V} \delta \chi\left(\mathbf{r}, t^{\prime}\right) e^{i\left(\mathbf{k}_{\mathbf{0}}-\mathbf{k}\right) \cdot \mathbf{r}} d^{3} r\right|^{2},
\end{gathered}
$$

where $\chi\left(\mathbf{r}, t^{\prime}\right)=\chi_{0}+\delta \chi\left(\mathbf{r}, t^{\prime}\right)$ (with $\left|\delta \chi\left(\mathbf{r}, t^{\prime}\right)\right| \ll\left|\chi_{0}\right|$ ) is the instantaneous linear susceptibility of the polarizable medium $\left(\chi_{0} \equiv \chi_{0}\left(\omega_{0}, \rho_{0}\right)\right)$. The time-dependent part, namely, $\delta \chi\left(\mathbf{r}, t^{\prime}\right)$, is generated by the acoustic vibration-induced local deformations of the elastic homogeneous medium, and $t$ is related to $t^{\prime}$ through the equation $\mathrm{c}\left(t-t^{\prime}\right)=|\mathbf{O P}-\mathbf{r}| . \mathbf{k} \approx k_{0} \mathbf{u}$ is the scattered wave-vector (with $\mathbf{u}=\mathbf{O P} /|\mathbf{O P}|=\mathbf{k} /|\mathbf{k}|$ ) and $\mathbf{q}=\mathbf{k}_{\mathbf{0}}-\mathbf{k}$ is the so-called exchanged wave-vector. In both equations, $\mathrm{A}$ is the same common factor. Let us remark that, if the rather crude Born approximation is relaxed, Equations (7) and (8) also hold within the quasistatic limit $\left(R / \lambda_{0} \ll 1\right)$. In this case, $\mathbf{k}_{\mathbf{0}}$ has to be suppressed in both the above equations, and in the explicit expression of $\mathrm{A}$, one has to replace $\left|\mathbf{E}_{0}\right|^{2}$ by the square modulus of the homogeneous local electric field in the sphere $\left(\mathbf{E}_{\text {int }}=3 /\left(\epsilon\left(\omega_{0}\right)+2\right) \mathbf{E}_{0}{ }^{35}\right)$.

For small scatterers $\left(R / \lambda_{0} \ll 1\right)$, the factor $e^{i \mathbf{q} \cdot \mathbf{r}}$, which will be referred to as "the retardation factor" in the following, can be replaced by 1 , showing that the Rayleigh scattering scales as $V^{2}$. This well-known result explains why, for subwavelength particles, the extinction cross-section is dominated by the absorption contribution which scales as $V^{35}$ and, concomitantly, why single-particle experiments aiming at measuring far-field Rayleigh scattering (dark-field microscopy) necessarily involve rather large particles (typically $\mathrm{D}=2 \mathrm{R}$ $>50 \mathrm{~nm})$. Since $\left|\delta \chi\left(\mathbf{r}, t^{\prime}\right)\right| \ll\left|\chi_{0}\right|$, and since $\delta \chi\left(\mathbf{r}, t^{\prime}\right)$ can take either positive or negative values in Equation (8), it seems surprising - at first sight - that the inelastic Raman scattering would obey a linear scaling law $I \propto V$, suggesting that the inelastic scattering could be the dominant process for nanosized particles.

From a microscopic point of view, the inelastic light scattering in metal particles stems from the time-varying changes of the core- and conduction electron-densities which are enslaved to, and follow the slow motions of the atoms around their equilibrium distances. Moreover, in the case of large enough particles ( $\mathrm{D} \gg$ a few $\mathrm{nms}$ ), a simple analysis allows to relate the vibrational frequencies and eigen-modes of the finite-size periodic lattice to the Lamb modes defined in the frame of the continuous medium approximation. This strongly suggests that the time-varying component of the macroscopic susceptibility, that is $\delta \chi(\mathbf{r}, t)$, is basically a function of the characteristics of the thermally populated Lamb modes (local density fluctuations, direction and amplitude of the displacement field, etc). In the present model, it is assumed that the local time-varying susceptibility fluctuations in the homogeneous medium are mainly related to those of the density $\rho$ (number of atoms/unit volume), as suggested, for instance, by the Clausius-Mossotti equation ${ }^{36}$ or the Drude theory of metals $\left(\chi_{\text {drude }}=-\omega_{p}^{2} /(\omega(\omega+i \Gamma))\right.$ with $\left.\omega_{p}^{2} \propto \rho^{35}\right)$. At room temperature the fluctuations of the density are indeed very tiny, so we have

$$
\begin{gathered}
\chi\left(\omega_{0}, \rho(\mathbf{r}, t)\right)=\chi\left(\omega_{0}, \rho_{0}+\delta \rho(\mathbf{r}, t)\right), \\
\left.\delta \chi\left(\omega_{0}, \mathbf{r}, t\right) \approx \frac{\partial \chi}{\partial \rho}\right|_{\rho_{0}} \delta \rho(\mathbf{r}, t),
\end{gathered}
$$

with $\delta \rho(\mathbf{r}, t)=\sum_{a} \delta \rho_{a}$, where the label a $\equiv(\ell, n, m)$ stands for any vibrational Lamb mode contributing to the inelastic Raman scattering.

Besides the relationship Equation (10), the second major feature of the present modeling consists in taking into account of on one hand the retardation factor and on the other hand 
the local electric field $\mathbf{E}(\mathbf{r})$ inside the particle. To our knowledge, both factors are systematically disregarded in previous works devoted to sub-wavelength particles. ${ }^{26}$ The spatial term in Equation (2) can be written as

$$
\begin{aligned}
I_{s}(a)= & \left.\omega_{0}^{4}\left|\frac{\partial \chi}{\partial \rho}\right|_{\rho_{0}}\right|^{2} \\
& \times\left|\mathbf{u} \times\left(\mathbf{u} \times\left(\int d^{3} r \mathbf{E}_{i n t}(\mathbf{r}) e^{-i \mathbf{k} \cdot \mathbf{r}} \frac{\partial \delta \rho_{a}(\mathbf{r})}{\partial Q_{a}}\right)\right)\right|^{2},
\end{aligned}
$$

where $\mathrm{Q}_{a}$ is the normal coordinate of the a-mode. In applying the continuity equation, namely, $\partial \rho / \partial t+\nabla \cdot(\rho \mathbf{V})=0$, where $\mathbf{V}$ is the velocity field, to each Lamb mode, and assuming the continuous medium approximation, one obtains

$$
\frac{\partial \delta \rho_{a}(\mathbf{r})}{\partial Q_{a}}=-\sqrt{\rho_{0}}\left[H(R-|\mathbf{r}|) \nabla \cdot \mathbf{u}_{\mathbf{a}}^{*}(\mathbf{r})-\delta(r-R) \mathbf{u}_{\mathbf{a}}^{*}(\mathbf{r}) \cdot \mathbf{e}_{\mathbf{r}}\right],
$$

where $\mathbf{u}_{\mathbf{a}}(\mathbf{r})$ is the normalized displacement field of the mode $\mathrm{a}\left(\sqrt{\int_{V} d^{3} r\left|\mathbf{u}_{\mathbf{a}}(\mathbf{r})\right|^{2}}=1\right)$ and $\mathbf{e}_{\mathbf{r}}$ the outward-pointing normal to the surface of the sphere. $H(x)$ is the Heaviside step function $(H(x)=1$ for $\mathrm{x}>0$ and $H(x)=0$ for $\mathrm{x}<0)$ and $\delta(x)$ the "Dirac function." A succinct derivation of this central model equation is given in the Appendix, and the present model will be fully described in great detail in a forthcoming paper. From the divergence theorem, it is straightforward to check the expected constraint

$$
\int_{V} \frac{\partial \delta \rho_{a}(\mathbf{r})}{\partial Q_{a}} d^{3} r=0
$$

which merely expresses the law of conservation of mass. The two terms on the right-hand side of Equation (12) are referred to as the "volume term" and "surface term," respectively, in the modeling. In the Lamb theory, ${ }^{34}$ the displacement fields of the vibrational modes a $\equiv(\ell, n, m)$ are specific linear combinations of the vector spherical harmonics $\mathbf{L}_{\ell, m}\left(\mathbf{r}, k_{L}\right)$, $\mathbf{M}_{\ell, m}\left(\mathbf{r}, k_{T}\right)$, and $\mathbf{N}_{\ell, m}\left(\mathbf{r}, k_{T}\right)$, where $k_{L}=\omega_{a} / c_{L}$ and $k_{T}=$ $\omega_{a} / c_{T}$ are the longitudinal and transverse wavevectors (these vector functions are solutions of the vector wave equation in the homogeneous medium). Equation (12) indicates that the torsional modes $(\propto \mathbf{M})$ are not Raman active (since $\nabla \cdot \mathbf{M}=0$ and $\left.\mathbf{e}_{\mathbf{r}} \cdot \mathbf{M}=0\right)$. So only the spheroidal modes $(\propto a \mathbf{L}+b \mathbf{N})$ are Raman active $\left(\nabla \cdot \mathbf{L} \neq 0, \mathbf{e}_{\mathbf{r}} \cdot \mathbf{L} \neq 0, \nabla \cdot \mathbf{N}=0\right.$, and $\left.\mathbf{e}_{\mathbf{r}} \cdot \mathbf{N} \neq 0\right)$, as also predicted by the existing BP and DID microscopic models. ${ }^{26}$

Equation (13) implies, if the local field in the particle is homogeneous, i.e., if the Born approximation is assumed, that the Raman intensity is zero in the absence of retardation effects. Actually, it should be stressed that, because of the finite light velocity, small inhomogeneities of the inner electric field always exist in small spherical particles. Nevertheless using the approximate result obtained in the quasistatic limit (i.e., $\mathbf{E}_{\text {int }}$ is homogeneous inside a small spherical- or ellipsoidalparticle) is quite justified for computing linear absorption and Rayleigh scattering. The $\mathbf{r}$-dependence of the product $\mathbf{E}_{\text {int }}(\mathbf{r}) e^{-i \mathbf{k} \cdot \mathbf{r}}$, which weights the individual wavelets scattered by each volume element of the particle, is one of the major ingredients of the present modeling of the inelastic scattering. From the analytical expression of the displacement field of the mode $a \equiv(\ell, n, m), \partial \delta \rho_{a}(\mathbf{r}) / \partial Q_{a}$ can be expressed as

$$
\frac{\partial \delta \rho_{a}(\mathbf{r})}{\partial Q_{a}}=-\sqrt{\rho_{0}}\left[g_{\ell, n}^{(V)}(r)+\delta(r-R) g_{\ell, n}^{(S)}(r)\right] Y_{\ell}^{m^{*}}(\theta, \phi) .
$$

Due to the orthogonality properties of the scalar spherical harmonics only the components of the product $\mathbf{E}_{\text {int }}(\mathbf{r}) e^{-i \mathbf{k} \cdot \mathbf{r}}$ of angular symmetry $Y_{\ell}^{m}(\theta, \phi)$ will contribute to the inelastic scattering of the mode a. An important technical part of the modeling consists in expanding analytically the weighting product factor on the scalar spherical harmonics. In particular, the internal electric field $\mathbf{E}_{\text {int }}(\boldsymbol{r})$ is expressed, thanks to the Mie theory, ${ }^{35}$ as a linear combination of the vector spherical harmonics $\mathbf{M}_{\ell^{\prime}, m^{\prime}}\left(\mathbf{r}, k_{1}\right)$ and $\mathbf{N}_{\ell^{\prime}, m^{\prime}}\left(\mathbf{r}, k_{1}\right)$, where $k_{1}=n_{1}\left(\omega_{0}\right) \omega_{0} / c$ is the optical wave-vector in the homogeneous medium $\left[n_{1}\right.$ is the particle index (a complex number in the case of metals)]. Actually the treatment of the angular part of the weighting factor can be handled analytically. Finally the radial integrals, which involve products of regular spherical Bessel functions $j_{n}(x)$, are evaluated numerically with a high accuracy.

\section{Results of the model}

Figs. 3(a) and 3(b) display the polarized Raman spectra obtained in the backscattering geometry for two spherical gold particles of diameters, respectively, $\mathrm{D}=18 \mathrm{~nm}$ and $\mathrm{D}=49 \mathrm{~nm}$, embedded in a transparent matrix of index $n_{m}=1.5$, with a $532 \mathrm{~nm}$ excitation wavelength. The complex index $n_{1}$ has been taken from the Johnson and Christie experimental data. ${ }^{37}$ Assuming that mechanical surface effects at the particle/matrix interface can be neglected, the Lamb modes of free spheres have been taken in our simulations (stress-free boundary conditions), using the sound velocities $c_{L}=3330 \mathrm{~m} / \mathrm{s}$ and

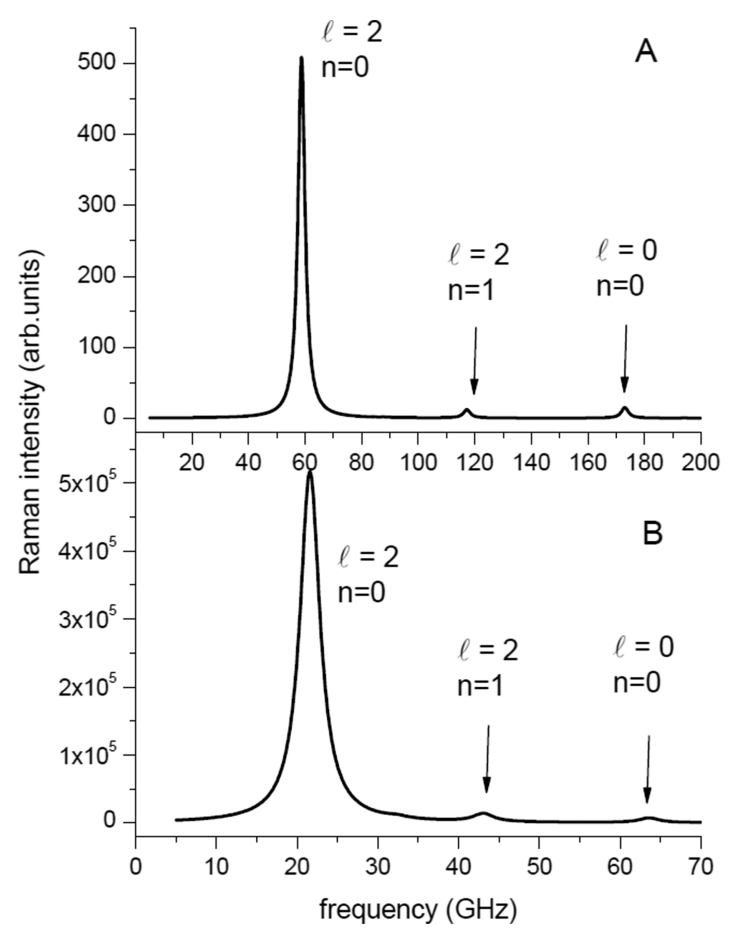

FIG. 3. Theoretical Raman spectra computed for two gold spheres ((a) radii $\mathrm{R}=9 \mathrm{~nm}$ and (b) $\mathrm{R}=24.5 \mathrm{~nm}$ ) embedded in a transparent matrix of index $n_{m}$ $=1.5$, for the $532 \mathrm{~nm}$ excitation wavelength and in the backscattering geometry. 
$c_{T}=1250 \mathrm{~m} / \mathrm{s}$. Note that each Raman peak is the sum of $2 \ell+1$ contributions because of the m-degeneracy of the Lamb modes (the frequency depends only on $\ell$ and $\mathrm{n}$ ). Finally each Raman peak computed in the present modeling has been convoluted with a Lorentzian-shape curve with a full width at half-maximum equal to $3 \mathrm{GHz}$ (arbitrary value). The location of the fundamental quadrupolar mode $(\ell=2, \mathrm{n}=0)$ agrees perfectly with the experimental findings. Because the inelastic Raman scattering is a very weak scattering process, and due to the poor signal-to-noise ratio, only this mode is conspicuous in the experimental spectra (see Figure 1), as well as in most experimental spectra reported in the literature. In the theoretical spectra, very weak contributions from the second harmonic of the $\ell=2$ sequence $(\ell=2, \mathrm{n}=1)$ and from the fundamental symmetric mode (the so-called breathing mode $\ell=0, \mathrm{n}=0$ ) are visible. Actually these features are quite general and have been observed in most simulations performed on gold and silver particles, for various conditions of excitation and/or detection. So the present modeling is able to promote, in the frame of the continuous medium approximation, the emergence of the quadrupolar and symmetric modes without resorting to symmetry-based selection rules ${ }^{25}$ or to a detailed atomistic description. ${ }^{26}$

Let us now analyze the size-dependence of the Raman intensity $I_{\text {Raman }}$ that is predicted in our modeling, noting that the calculated intensity ratio $I_{A u 49}^{c a l c} / I_{A u 18}^{c a l c} \approx 20 \times 50$ is about 50 times larger than the volume ratio $V_{A u 49} / V_{A u 18} \approx 20$ (see Figure 3). In Equation (4), the factor $\left(1+n\left(\omega_{a}\right)\right) / \omega_{a}$ scales as $R^{2}$ at room temperature, and the coupling coefficient $C\left(\omega_{a}\right)$ of the atomistic BP and DID microscopic models ${ }^{26}$ scales as R, leading to a net size dependence $I_{\text {Raman }} \propto R^{3} \propto V_{N P}$. As a matter of fact, if the volume term or the surface term is set equal to zero [i.e., $g_{\ell, n}^{(V)}(r)=0$ or $g_{\ell, n}^{(S)}(r)=0$ in Equation (14)], the spatial term $I_{s}(a)$, namely, the mode-radiation coupling coefficient $C\left(\omega_{a}\right)$ of the present theory, scales as $\mathrm{R}$ when the weighting factor is neglected, as in existing models (this property is exhibited by the analytical expressions of both radial functions). However, in the present modeling, the Raman intensity is proportional to the square modulus of the weighted algebraic sum of two contributions which compensate one anotherperfectly - if both the retardation factor and the inhomogeneity of the inner electric field are disregarded. The departure from this exact cancellation for finite radii leads to an effective volume dependence — not easy to predict a priori—differing from the previous linear law, and expected to be more in line with the qualitative analysis inferred from Equations (7) and (8). Figure 4 shows the size dependence that is predicted in the present theory for the fundamental quadrupolar mode, using the excitation and detection conditions described previously, in the size range $[\mathrm{R}=5 \mathrm{~nm} \leftrightarrow \mathrm{R}=50 \mathrm{~nm}]$. A scaling law $I_{\text {Raman }} \propto V^{2.3}$ is obtained.

It should be noted that the present modeling leads to an overestimation of the experimental size dependence of the Raman intensity ratio. We think that the multiple scattering and reabsorption of Stokes and anti-Stokes photons in the metal, which are not included in the model (as also in existing atomistic models ${ }^{26,28}$ ), are responsible for this discrepancy. A thorough analysis of these processes, which are probably not easy to quantify accurately in the present context which

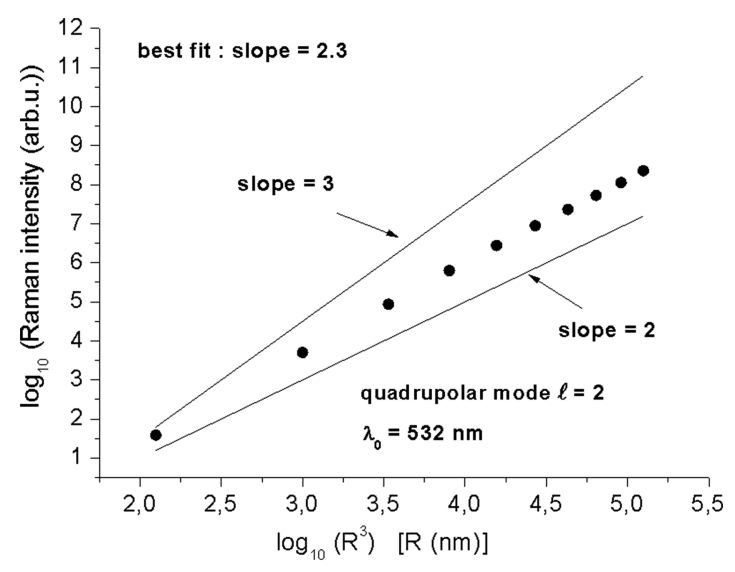

FIG. 4. Evolution of the Raman intensity (arbitrary units) as a function of the particle volume, for the fundamental quadrupolar mode.

involves a finite system, is out of scope of this paper. Nevertheless it is possible to consider some reasonable qualitative indications. Besides the depolarization effect, multiple scattering in the particle is expected to modify the angular distribution of the Stokes and anti-Stokes photons, and probably will affect also the size dependency. Since the optical frequencies $\omega_{0} \pm \omega_{a}$ are very close to each other, it is expected that, for a given size, the multiple scattering events will impact all the Raman lines in the same way, preserving therefore the overall Raman spectrum (simple multiplicative factor). In contrast, it is clear that the reabsorption processes will impact strongly the size dependence of the Raman intensity ratio. Assuming that this reabsorption process obeys the same scaling law as in the Mie theory, the rate of detected Stokes and anti-Stokes photons has to be reduced by a factor proportional to $V_{N P}$, at least for small sizes. If it is the case the predicted Raman intensity ratio would be close to the experimental findings. Finally let us point out that a stronger disagreement between the experiment and atomistic BP and DID models would result in including the reabsorption processes in the complete theoretical modeling.

\section{SUMMARY}

To conclude, we have experimentally investigated the dependence of the Raman intensity from NP Lamb modes upon the volume of the NPs using resonant LF Raman spectroscopy. Our data clearly point out strong differences with prediction from microscopic models when the NPs are metallic and when the light is tuned in resonance with their dipolar surface plasmon. To explain the observed discrepancies, a simple model of Raman scattering has been introduced, based on the density fluctuations in the NPs produced by the vibrations. Our model provides a unified picture for any material and is suitable for handling both small and large size ranges, as well as non-resonant and resonant excitation conditions in the case of metallic species. Our discussion is focused here on the simple case of non-interacting NPs but opens up perspective in understanding NP LF spectra in more complex situations such as recently evidenced in the case of dimers of metallic NPs ${ }^{4}$ or interacting metallic NPs/substrate. ${ }^{9}$ 


\section{ACKNOWLEDGMENTS}

These studies were supported by the ANR Nanovip project, Grant No. ANR-13-JS10-0002 of the French Agence Nationale de la Recherche. We thank the Centre Technologique des Microstructures at Villeurbanne $(\mathrm{CT} \mu)$ for access to the microscope platform for TEM characterizations.

\section{APPENDIX: RELATIONSHIP LINKING THE INDUCED-DENSITY FLUCTUATION AND NORMAL COORDINATE OF THE VIBRATIONAL A-MODE [DERIVATION OF EQUATION (12)]}

The building up of the macroscopic classical modeling presented in this work was motivated by the following observations. First, the classical Mie theory suitably describes the optical properties, in particular the elastic Rayleigh scattering, of isotropic homogeneous objects described within the continuous medium approximation and is applicable to any kind of materials. Moreover, in the case of metallic species which can sustain collective electronic excitations in narrow spectral ranges, the off-resonance and resonance situations are treated with an equal footing thanks to a common formalism. Second, in the frame of the continuous medium approximation too, the Lamb theory for an elastic isotropic sphere suitably predicts the low-frequency vibrational modes that are observed in Raman spectroscopy experiments on small and large spherical particles, for both dielectric and metallic species.

These preliminary remarks strongly suggest that, in the context of inelastic Raman or Brillouin spectroscopies, a unique macroscopic classical formalism should exist for dealing with any material described within the continuous medium approximation and for handling both resonant and non-resonant conditions in the case of metallic species.

Up to now, different approaches, in general atomistic (that is, retaining the discrete nature of the atomic background), have been introduced, and various systematic approximations have been assumed implicitly. For small particles (size $<$ excitation wavelength), retardation effects are neglected: the electric field is assumed strictly homogeneous in the entire particle volume (this amounts to assuming —implicitly—-the Born approximation or the strict quasistatic approximation) and the retardation factor $\left(\mathrm{e}^{i \mathbf{q} \cdot \mathbf{r}} \approx 1\right)$ is neglected.

For large particles, the retardation factor is included in the modeling but the inhomogeneity of the internal electric field inside the particle is not taken into account. So acoustic modes of high $\ell$ values are observed only for particle sizes on the order of, or exceeding, the wavelength of the irradiating electric field.

To our knowledge, in the case of small particles, the application of the classical Raman/Brillouin intensity formula (which is basically derived from the classical theory of a radiating point-dipole described in textbooks), taking into account-without any numerical approximation-both ingredients (namely, the retardation factor and the inhomogeneity of the internal field), was never reported in the literature. Since our modeling is based on standard classical electrodynamics equations and on the classical Raman scattering theory, only the derivation of the Raman factor (Eq. (12)) deserves to be outlined. We have thus to express the density fluctuation in function of the vibrational normal mode coordinates $Q_{a}$ of the particle.

Let us consider a spherical homogeneous particle of volume $V$ containing $N$ atoms, of average density $\rho_{0}=N / V$, and let us assume a simple Bravais lattice (one atom in each primitive cell) since this case corresponds to noble metals (fcc structure). Within the harmonic approximation, the vibrational motion of the discrete atomic lattice can be described through those of its $3 N-6$ normal mode coordinates $\mathrm{Q}_{a}$, each of them corresponding to a one-dimensional harmonic oscillator (frequency $\omega_{a}$ ) in the expression of the vibrational Hamiltonian. These normal coordinates are related to the atomic displacements $u_{i, \alpha}$ about their equilibrium positions $\mathbf{r}_{i}^{e q}$ (i denotes the atomic site in the lattice and $\alpha$ the Cartesian coordinate, $\mathrm{x}, \mathrm{y}$, or z) through a square unitary matrix $M$ of dimension $3 N-6$, namely,

$$
\begin{gathered}
Q_{a}=\sum_{i, \alpha} M_{a, i, \alpha} u_{i, \alpha}, \\
u_{i, \alpha}=\sum_{a}\left[M^{-1}\right]_{i, \alpha, a} Q_{a}=\sum_{a} M_{a, i, \alpha}^{*} Q_{a} .
\end{gathered}
$$

Since the matrix $\mathrm{M}$ is unitary, the dimensionless matrix elements satisfy the following properties:

$$
\begin{gathered}
\sum_{i, \alpha}\left|M_{a, i, \alpha}\right|^{2}=1 \quad \forall a, \\
\sum_{i, \alpha} M_{a, i, \alpha} M_{a^{\prime}, i, \alpha}^{*}=0 \quad\left(a \neq a^{\prime}\right) .
\end{gathered}
$$

As in molecular physics, the matrix coefficients $M_{a, i, \alpha}$ reflect, for any given normal coordinate $Q_{a}$, the relative atomic displacements (amplitude and algebraic direction) at each atomic site of the lattice. For large enough particles (large $N$ ), each vibrational normal mode coordinate of the discrete lattice can be associated with a specific Lamb mode $a \equiv(\ell, m, n)$ [obviously only the Lamb modes of low indexes $n$ and $\ell$ have to be considered (the maximum values depend on $N$ )]. We have thus

$$
M_{a, i, \alpha}=\beta_{a} u_{a, \alpha}\left(\mathbf{r}=\mathbf{r}_{i}^{e q}\right),
$$

where $u_{a, \alpha}\left(\mathbf{r}=\mathbf{r}_{i}^{e q}\right)$ is the Cartesian component $\alpha$ of the displacement field $\mathbf{u}_{a}$ of the corresponding Lamb mode $a$ $\equiv(\ell, m, n)$, and $\beta_{a}$ a real constant factor (unit length ${ }^{-1}$ ) to be determined.

Assuming that $u_{a, \alpha}\left(\mathbf{r}=\mathbf{r}_{i}^{e q}\right)$ varies slowly relative to the linear size of the primitive cell, Equation (A3) can be written as follows within the continuous medium approximation:

$$
\rho_{0} \beta_{a}^{2} \int_{V} d^{3} r\left|\mathbf{u}_{a}(\mathbf{r})\right|^{2}=1
$$

We have therefore

$$
\beta_{a}=\frac{1}{\sqrt{\rho_{0}}} \frac{1}{\sqrt{\int_{V} d^{3} r\left|\mathbf{u}_{a}(\mathbf{r})\right|^{2}}} .
$$

From Equations (A2), (A5), and (A7), the atomic displacements can be expressed as

$$
\mathbf{u}_{i}(t)=\frac{1}{\sqrt{\rho_{0}}} \sum_{a} \frac{\mathbf{u}_{a}^{*}\left(\mathbf{r}=\mathbf{r}_{i}^{e q}\right)}{\sqrt{\int_{V} d^{3} r\left|\mathbf{u}_{a}(\mathbf{r})\right|^{2}}} Q_{a}(t)
$$


with $Q_{a}(t)=Q_{a} e^{i \omega_{a} t}$. Within the continuous medium approximation, (A8) can be written as

$\mathbf{u}(\mathbf{r}, t)=\sum_{a} \beta_{a} \mathbf{u}_{a}^{*}(\mathbf{r}) Q_{a}(t)=\frac{1}{\sqrt{\rho_{0}}} \sum_{a} \frac{\mathbf{u}_{a}^{*}(\mathbf{r})}{\sqrt{\int_{V} d^{3} r\left|\mathbf{u}_{a}(\mathbf{r})\right|^{2}}} Q_{a}(t)$.

The quantity $\frac{\partial \delta \rho(\mathbf{r})}{\partial Q_{a}}$ is then obtained in using the continuity equation, namely,

$$
\frac{\partial}{\partial t} \rho(\mathbf{r}, t)+\nabla \cdot(\rho(\mathbf{r}, t) \mathbf{V}(\mathbf{r}, t))=0,
$$

where $\rho(\mathbf{r}, t)$ is the local density and $\mathbf{V}(\mathbf{r}, t)$ the velocity field. We have

$$
\rho(\mathbf{r}, t)=\rho_{0} H(R-|\mathbf{r}|)+\sum_{a} \delta \rho_{a}(\mathbf{r}, t),
$$

where $\delta \rho_{a}(\mathbf{r}, t)=\delta \rho_{a}(\mathbf{r}) e^{i \omega_{a} t}$,

$$
V(\mathbf{r}, t)=\sum_{a} \beta_{a} \mathbf{u}_{a}^{*}(\mathbf{r}) \frac{\partial}{\partial t} Q_{a}(t) .
$$

Keeping only the first-order terms (linear regime $\equiv$ weak perturbation), Equation (A10) can be written as

$$
\frac{\partial}{\partial t} \sum_{a} \delta \rho_{a}(\mathbf{r}, t)+\nabla \cdot\left(\rho_{0} H(R-|\mathbf{r}|) \sum_{a} \beta_{a} \mathbf{u}_{a}^{*}(\mathbf{r}) \frac{\partial}{\partial t} Q_{a}(t)\right)=0 .
$$

For each normal (Lamb) mode, one obtains finally the desired relationship relating the density fluctuation to the normal mode coordinate $Q_{a}$,

$$
\begin{gathered}
\delta \rho_{a}(\mathbf{r})=-\beta_{a} \nabla \cdot\left(\rho_{0} H(R-|\mathbf{r}|) \mathbf{u}_{a}^{*}(\mathbf{r})\right) Q_{a}, \\
\frac{\partial \delta \rho_{a}(\mathbf{r}, t)}{\partial Q_{a}}=-\beta_{a} \rho_{0}\left(H(R-|\mathbf{r}|) \nabla \cdot \mathbf{u}_{a}^{*}(\mathbf{r})-\delta(r-R) \mathbf{u}_{a}^{*}(\mathbf{r}) \cdot \mathbf{e}_{r}\right) .
\end{gathered}
$$

Equation (12) of the main text is thus established. It is worthwhile interpreting physically the surface term entering Equation (A15) (term involving the Dirac function) from a simple pictorial arguing. The derivation of the continuity equation is mostly derived in considering a small cubic volume and equating the rate of change of the mass contained in this small cube with the mass flow crossing (from the outside) the six faces of the cube. Actually, caution has to be taken when the continuity equation is applied in the close vicinity of the particle boundary since no mass flow comes from the exterior. Writing the static particle density as $\rho(\mathbf{r})=\rho_{0} H(R-|\mathbf{r}|)$ allows to suppress automatically the spurious mass flow crossing the particle surface. As pointed out in the main text, the presence of the surface term is necessary to ensure the law of conservation of mass (Equation (13)).
${ }^{1}$ G. Bachelier, J. Margueritat, A. Mlayah, J. Gonzalo, and C. Afonso, Phys. Rev. B 76, 235419 (2007).

${ }^{2}$ J. Margueritat, J. Gonzalo, C. N. Afonso, A. Mlayah, D. B. Murray, and L. Saviot, Nano Lett. 6, 2037 (2006).

${ }^{3}$ N. Large, L. Saviot, J. Margueritat, J. Gonzalo, C. N. Afonso, A. Arbouet, P. Langot, A. Mlayah, and J. Aizpurua, Nano Lett. 9, 3732 (2009).

${ }^{4}$ A. Girard, L. Saviot, S. Pedetti, M. D. Tessier, J. Margueritat, H. Gehan, B. Mahler, B. Dubertret, and A. Mermet, Nanoscale 8, 13251 (2016).

${ }^{5}$ H. Portales, N. Goubet, L. Saviot, S. Adichtchev, D. B. Murray, A. Mermet, E. Duval, and M.-P. Pileni, Proc. Natl. Acad. Sci. U. S. A. 105, 14784 (2008).

${ }^{6}$ H. Portales, N. Goubet, S. Sirotkin, and E. Duval, Nano Lett. 12, 5292 (2012).

${ }^{7}$ H. Portales, N. Goubet, L. Saviot, P. Yang, S. Sirotkin, E. Duval, A. Mermet, and M.-P. Pileni, ACS Nano 4, 3489 (2010).

${ }^{8}$ A. Crut, P. Maioli, N. Del Fatti, and F. Vallée, Ultrasonics 56, 98 (2015).

${ }^{9}$ S. Tripathy, R. Marty, V. K. Lin, S. L. Teo, E. Ye, A. Arbouet, L. Saviot, C. Girard, M. Y. Han, and A. Mlayah, Nano Lett. 11, 431 (2011).

${ }^{10}$ M. H. Kuok, H. S. Lim, S. C. Ng, N. N. Liu, and Z. K. Wang, Phys. Rev. Lett. 90, 255502 (2003).

${ }^{11}$ M. Mattarelli, M. Montagna, T. Still, D. Schneider, and G. Fytas, Soft Matter 8, 4235 (2012).

${ }^{12}$ L. Saviot, D. Machon, A. Mermet, D. B. Murray, S. Adichtchev, J. Margueritat, F. Demoisson, M. Ariane, and M. d. C. Marco de Lucas, J. Phys. Chem. C 116, 22043 (2012).

${ }^{13}$ L. Saviot, D. Machon, L. Debbichi, A. Girard, J. Margueritat, P. Krüger, M. C. Marco de Lucas, and A. Mermet, J. Phys. Chem. C 118, 10495 (2014).

${ }^{14}$ S. Huang, L. Liang, X. Ling, A. A. Puretzky, D. B. Geohegan, B. G. Sumpter, J. Kong, V. Meunier, and M. S. Dresselhaus, Nano Lett. 16, 1435 (2016).

${ }^{15}$ Y. Zhao, X. Luo, H. Li, J. Zhang, P. T. Araujo, C. K. Gan, J. Wu, H. Zhang, S. Y. Quek, M. S. Dresselhaus, and Q. Xiong, Nano Lett. 13, 1007 (2013).

${ }^{16}$ B. Dacosta Fernandes, M. Spuch-Calvar, H. Baida, M. Tréguer-Delapierre, J. Oberlé, P. Langot, and J. Burgin, ACS Nano 7, 7630 (2013).

${ }^{17}$ H. Lange, M. Mohr, M. Artemyev, U. Woggon, and C. Thomsen, Nano Lett. 8, 4614 (2008).

${ }^{18}$ J. H. Kim, H. Kim, H. Rho, Y.-J. Choi, and J.-G. Park, J. Phys. D: Appl. Phys. 47, 135104 (2014).

${ }^{19}$ D. B. Murray and L. Saviot, Phys. Rev. B 69, 094305 (2004).

${ }^{20}$ L. Saviot, D. B. Murray, E. Duval, A. Mermet, S. Sirotkin, and M. d. C. Marco de Lucas, Phys. Rev. B 82, 115450 (2010).

${ }^{21}$ M. Kanehisa, Phys. Rev. B 72, 241405 (2005).

${ }^{22}$ M. Kanehisa, Phys. Rev. B 74, 197402 (2006).

${ }^{23}$ Y. Li, H. S. Lim, S. C. Ng, Z. K. Wang, and M. H. Kuok, Chem. Phys. Lett. 461, 111 (2008).

${ }^{24}$ M. Mattarelli and M. Montagna, Chem. Phys. Lett. 524, 112 (2012).

${ }^{25}$ E. Duval, Phys. Rev. B 46, 5795 (1992).

${ }^{26}$ M. Montagna and R. Dusi, Phys. Rev. B 52, 10080 (1995).

${ }^{27}$ T. Still, M. Mattarelli, D. Kiefer, G. Fytas, and M. Montagna, J. Phys. Chem. Lett. 1, 2440 (2010).

${ }^{28}$ M. Montagna, Phys. Rev. B 77, 045418 (2008).

${ }^{29}$ A. Girard, H. Gehan, A. Crut, A. Mermet, L. Saviot, and J. Margueritat, Nano Lett. 16, 3843 (2016).

${ }^{30}$ S. Marhaba, G. Bachelier, C. Bonnet, M. Broyer, E. Cottancin, N. Grillet, J. Lermé, J.-L. Vialle, and M. Pellarin, J. Phys. Chem. C 113, 4349 (2009).

${ }^{31}$ J. Lermé, J. Phys. Chem. C 119, 21087 (2015).

${ }^{32}$ P. Nordlander, C. Oubre, E. Prodan, K. Li, and M. I. Stockman, Nano Lett. 4, 899 (2004).

${ }^{33}$ H. Lamb, Proc. London Math. Soc. 13, 189 (1882).

${ }^{34}$ A. C. Eringen and E. S. Suhubi, Elastodynamics (Academic Press Inc., 1975), Vol. 2, pp. 804-833.

${ }^{35}$ C. F. Bohren and D. P. Huffman, Absorption and Scattering of Light by Small Particles (Wiley, NewYork, 1983).

${ }^{36}$ J. D. Jackson, Classical Electrodynamics, 3rd ed. (John Wiley \& Sons, Inc., New York, 1998).

${ }^{37}$ P. B. Johnson and R. W. Christy, Phys. Rev. B 6, 4370 (1972). 